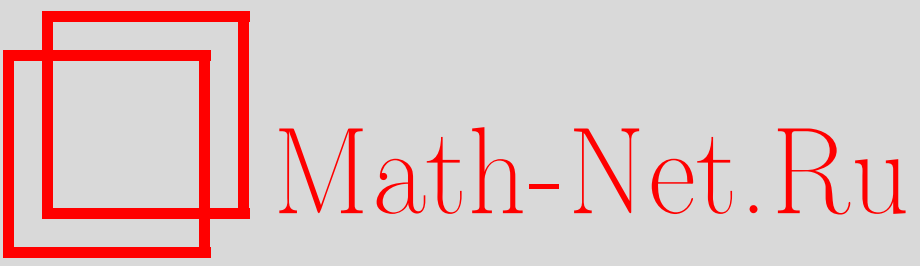

А. М. Чеботарев, Априорные оценки для квантовых динамических полугрупп, TMФ, 2003, том 134, номер 2, 185-190

DOI: https://doi.org/10.4213/tmf148

Использование Общероссийского математического портала Math-Net.Ru подразумевает, что вы прочитали и согласны с пользовательским соглашением

http://www.mathnet.ru/rus/agreement

Параметры загрузки:

IP : 54.157 .27 .8

26 апреля 2023 г., $07: 06: 51$ 


\section{АПРИОРНЫЕ ОЦЕНКИ ДЛЯ КВАНТОВЫХ ДИНАМИЧЕСКИХ ПОЛУГРУПП}

Получены априорные оценки для решений квантовых уравнений марковской эволюции в представлениях Гейзенберга и Шредингера.

Ключевые слова: квантовая динамическая полугруппа, уравнение марковской эволюции, априорные оценки минимальной квантовой динамической полугруппы.

Пусть $\mathcal{H}$ - сепарабельное гильбертово пространство с полуторалинейным скалярным произведением $(\cdot, \cdot)$ и $\mathcal{B}(\mathcal{H})$ - алгебра фон Неймана, состояшая из всех ограниченных операторов, действуюших в $\mathcal{H}$. Рассмотрим формальный генератор

$$
\mathcal{L}(B)=\Phi(B)-\frac{1}{2} \Phi(I) B-\frac{1}{2} B \Phi(I)+i[H, B], \quad \Phi(B)=\sum_{k} \Phi_{k}^{*} B \Phi_{k},
$$

квантовой динамической полугруппы (КДП) $P_{t}(B)=e^{t \mathcal{L}}(B)$ на алгебре $\mathcal{B}(\mathcal{H})$. Предполагается, что коэффишиентами генератора $\mathcal{L}$ являются плотно определенный симметричный оператор $H$ (гамильтониан, действуюший в гильбертовом пространстве $\mathcal{H}$ ) и нормальное вполне положительное отображение $\Phi$ (диссипативная часть, действуюшая в алгебре $\mathcal{B}(\mathcal{H})$ ). Структура генератора $\mathcal{L}$ сходна со структурой классического уравнения Колмогорова-Феллера. Аналогично классическому случаю уравнение марковской эволюции

$$
\frac{d}{d t} P_{t}(B)=\mathcal{L}\left(P_{t}(B)\right), \quad P_{0}(B)=B,
$$

при определенных условиях имеет минимальное решение, называемое минимальной $K Д \Pi[1]-[3]$. КДП в представлении Гейзенберга $P_{t}$ и КДП в представлении Шредингера $T_{t}$ двойственны относительно скалярного произведения $\left\langle T_{t}(\rho), B\right\rangle=\operatorname{Tr}\left\{T_{t}(\rho) B\right\}=$ $\operatorname{Tr}\left\{\rho P_{t}(B)\right\}=\left\langle\rho, P_{t}(B)\right\rangle$, где $\rho$ - элемент алгебры $\mathcal{T}(\mathcal{H})$ операторов с конечным следом, а $B$ - элемент алгебры $\mathcal{B}(\mathcal{H})$ ограниченных операторов. Очевидно, что если $P_{t}(I)=I$, то $\operatorname{Tr} T_{t}(\rho)=\operatorname{Tr} \rho$. Пусть $\Lambda$ и $\Phi_{t}(I)$ - самосопряженные положительные операторы такие, что $\Lambda \geqslant \Phi_{t}(I) \geqslant 0$, и $-G=-\Phi(I) / 2-i H$ - генератор сильно непрерывной сжимающей полугруппы $W_{t}=e^{-t G}$ в $\mathcal{H}$. Не теряя обшности, будем считать, что для $\Lambda$

* Московский государственный университет, Москва, Россия. E-mail: alex@chebotar.phys.msu.su 
существует ограниченный обратный оператор. Ниже получены условия, при которых выполняется неравенство

$$
\operatorname{Tr}\left\{B T_{t}(\rho)\right\} \leqslant e^{c t}\left\|\Lambda^{-1 / 2} B \Lambda^{-1 / 2}\right\| \operatorname{Tr}\left(\Lambda^{1 / 2} \rho \Lambda^{1 / 2}\right) .
$$

Неравенства такого вида важны для оценки погрешности численного решения уравнений марковской эволюции методом квантовых траекторий с переменным конечным базисом [4], [5].

Обозначим через $\mathcal{H}_{k} \subset \mathcal{H}, k=0,1,2$, гильбертовы пространства со скалярными произведениями $(g, h)_{k}=\left(\Lambda^{k / 2} g, \Lambda^{k / 2} h\right)$.

ЛЕмма 1. Если существует постоянная $с>0$ такая, что

$$
-2 \operatorname{Re}\left(\Lambda^{k / 2} G \psi, \Lambda^{k / 2} \psi\right) \leqslant c\left\|\Lambda^{k / 2} \psi\right\|^{2}
$$

для любого $\psi \in \mathcal{H}_{k}, k=1,2$, то полугруппа $W_{t}$ ограничена и сильно непрерьвна в $\mathcal{H}_{k}$.

ДокАЗАТЕЛЬСтво. Пусть $\psi$ - решение резольвентного уравнения $(\lambda G+I) \psi=\varphi$ и $\varphi \in \mathcal{H}_{k}$, т.е. $\varphi=\Lambda^{-k / 2} \theta, \theta \in \mathcal{H}$. Тогда $\left\|\Lambda^{k / 2}(\lambda G+I) \psi\right\|^{2}=\|\theta\|^{2}$, и в силу оценки (2) имеем $2 \operatorname{Re}\left(\Lambda^{k / 2} G \psi, \Lambda^{k / 2} \psi\right) \geqslant-c\left\|\Lambda^{k / 2} \psi\right\|^{2}$. Поэтому при всех $\lambda>2 c$ выполняется неравенство

$\|\theta\|^{2}=\left\|\Lambda^{k / 2}(\lambda G+I) \psi\right\|^{2} \geqslant(1-2 \lambda c)\left\|\Lambda^{k / 2} \psi\right\|^{2}=(1-2 \lambda c)\left\|\Lambda^{k / 2}(\lambda G+I)^{-1} \Lambda^{-k / 2} \theta\right\|^{2}$.

Следовательно,

$$
\left\|\left(I+\frac{G t}{N}\right)^{-1}\right\|_{k}=\left\|\left(I+\frac{G_{k} t}{N}\right)^{-1}\right\| \leqslant\left(1-\frac{2 c t}{N}\right)^{-1 / 2},
$$

где $G_{k}=\Lambda^{k / 2} G \Lambda^{-k / 2}$. Учитывая, что

$$
W_{t}=\underset{N \rightarrow \infty}{\mathrm{S}-\lim }\left(I+\frac{G t}{N}\right)^{-N}
$$

получаем

$$
\begin{aligned}
\left\|\Lambda^{k / 2} W_{t} \Lambda^{-k / 2}\right\| & =\lim _{N \rightarrow \infty}\left\|\left(I+\frac{G_{k} t}{N}\right)^{-N}\right\| \leqslant\left\|\left(I+\frac{G_{k} t}{N}\right)\right\|^{N} \leqslant \\
& \leqslant \lim _{N \rightarrow \infty}\left(1-\frac{2 c t}{N}\right)^{-N / 2}=e^{c t}
\end{aligned}
$$

что доказывает ограниченность полугруппы $W_{t}$ в $\mathcal{H}_{k}$. Попутно можно заметить, что оценка нормы резольвентного оператора $(I+\lambda G)^{-1}$ в $\mathcal{H}_{k}$ совпадает с оценкой нормы резольвентного оператора $\left(I+\lambda G_{k}\right)^{-1}$ в $\mathcal{H}$.

Нетрудно видеть, что поскольку оператор $\Lambda$ замкнут и ограниченно обратим, а оператор $G \Lambda^{-k / 2}$ замкнут, то $G_{k}$ также замкнут в $\mathcal{H}$ (см. [6], гл. III, $\S 5$, п. 2, задача 5.12). 
Таким образом, оператор $-G_{k}$ удовлетворяет условиям теоремы о генераторе квазиограниченной сильно непрерьвной полугруппы $W_{t}^{(k)}=\Lambda^{k / 2} W_{t} \Lambda^{-k / 2}=e^{-t \Lambda^{k / 2} G \Lambda^{-k / 2}}$, действуюшей в $\mathcal{H}$ (см. [6], гл. IX, $\S 1$, п. 4).

Поскольку полугруппа $W_{t}$ ограничена в $\mathcal{H}_{k}$, то достаточно доказать ее непрерывность на любом плотном подмножестве, например на $\mathcal{H}_{\mu, k}=\Lambda^{k / 2}(\mu G+I)^{-N} \Lambda^{-k / 2} \mathcal{H} \subset$ $\mathcal{H}$. Пусть $h \in \mathcal{H}_{\text {и }} \psi=\Lambda^{k / 2}(\mu G+I)^{-N} \Lambda^{-k / 2} h \in \mathcal{H}_{\mu, k}$. Тогда $\left\|\Lambda^{k / 2}\left(W_{t}-I\right) \Lambda^{-k / 2} \psi\right\|=$ $\left\|\left(W_{t}^{(k)}-I\right) \psi\right\|$. Теперь сильная непрерывность $W_{t}$ в $\mathcal{H}_{k}$ следует из сильной непрерывности $W_{t}^{(k)}$ в $\mathcal{H}$. Лемма доказана.

ЛЕмма 2. Предположим, что условие (2) выполнено. Если

$$
A_{k, n}=\Lambda^{(k-1) / 2} \Phi_{n} \Lambda^{-k / 2} \in \mathcal{B}(\mathcal{H}), \quad \sum_{n} A_{k, n}^{*} A_{k, n} \in \mathcal{B}(\mathcal{H}), \quad k=1,2,
$$

то квадратичная форма $f_{t, s}=\Phi\left(P_{s}\right)\left[W_{t-s} \psi\right], \psi \in \mathcal{H}_{k}$, ограничена и непрерывна по $t$ us для любого ограниченного и сильно непрерывного в $\mathcal{H}_{k-1}$ семейства операторов $P_{t}$.

ДокАЗАТЕЛЬСтво. Учитывая, что $\Phi(X)=\sum_{n} \Phi_{n}^{*} X \Phi_{n}$, запишем квадратичную форму $f_{t, s}$ как сумму $\sum_{n}\left(h_{k}(t, s), B_{k, n}(t) h_{k}(t, s)\right)$, где

$$
\begin{gathered}
h_{k}(t, s)=W_{t-s}^{(k)} h, \quad W_{t}^{(k)}=\Lambda^{k / 2} W_{t} \Lambda^{-k / 2}, \quad \psi=\Lambda^{-k / 2} h, \quad h \in \mathcal{H}, \\
A_{k, n}=\Lambda^{(k-1) / 2} \Phi_{n} \Lambda^{-k / 2} \in \mathcal{B}(\mathcal{H}), \\
B_{k, n}(t)=A_{k, n}^{*} \Lambda^{-(k-1) / 2} P_{t} \Lambda^{-(k-1) / 2} A_{k, n} \in \mathcal{B}(\mathcal{H}) .
\end{gathered}
$$

Поскольку операторы $W_{t-s}^{(k)}$ ограничены и сильно непрерывны в $\mathcal{H}$, то векторы $h_{k}(t, s)$ непрерывны по норме $\mathcal{H}$ по переменным $t$ и $s$, и в сумме

$$
\begin{aligned}
\left|f_{t, s}-f_{\tau, s}\right| \leqslant & \sum_{n}\left\{\left(\left\|h_{k}(t, s)\right\|+\left\|h_{k}(\tau, s)\right\|\right)\left\|B_{k, n}(s)\right\|\left\|h_{k}(t, s)-h_{k}(\tau, s)\right\|+\right. \\
& \left.+\left\|h_{k}(t, s)\right\|\left\|\left(B_{k, n}(t)-B_{k, n}(\tau)\right) h_{k}(t, s)\right\|\right\}
\end{aligned}
$$

каждое слагаемое сходится к нулю при $\tau \rightarrow t$. Заметим также, что имеет место неравенство

$$
f_{k}(t, s) \leqslant\left\|B_{t}\right\| \sum_{n}\left(W_{t-s}^{(k)} h, A_{k, n}^{*} A_{k, n} W_{t-s}^{(k)} h\right),
$$

где по условию ряд $\sum_{n} A_{k, n}^{*} A_{k, n}$ сильно сходится к ограниченному оператору. Поскольку сильная сходимость равномерна на компактных множествах, а множество векторов $\left\{W_{t-s}^{(k)} h, s \leqslant t, t \in T\right\}$ при фиксированном $h$ компактно для любого компакта $T \in$ $\mathbb{R}_{+}$, то в силу приведенного выше неравенства по теореме Лебега о предельном переходе в сумме возможен почленный предельный переход при $\tau \rightarrow t$. Лемма доказана.

Предположим, что для любого $\psi \in \operatorname{dom} \Lambda$ выполнено следуюшее условие относительной ограниченности [2], [3]:

$$
\sum_{k}\left\|\Lambda^{1 / 2} \Phi_{k} \psi\right\|^{2}-2 \operatorname{Re}\left(\Lambda^{1 / 2} G \psi, \Lambda^{1 / 2} \psi\right) \leqslant c\left\|\Lambda^{1 / 2} \psi\right\|^{2},
$$

где $c \geqslant 0$. 
ТЕОРЕмА. Если выполнены предположсения лемм 1, 2 и условие (4), а оператор Н симметричен на $\operatorname{dom} \Lambda$, то для минимальной КДП выполнена априорная оченка

$$
P_{t}^{\min }(\Lambda) \leqslant \Lambda e^{c t}
$$

ДоказАТЕЛЬСТво. Через $\operatorname{CPn}(\mathcal{H})$ мы обозначаем конус ограниченных вполне положительных нормальных отображений, действуюших в алгебре наблюдаемых $\mathcal{B}(\mathcal{H})[7]$, [8]. Если выполнены предположения лемм 1, 2, то для любого ограниченного сильно непрерывного семейства операторов $X_{s}$ семейство квадратичных форм

$$
\Phi_{*}\left(X_{s}\right)\left[W_{t-s} \psi\right]=A_{*}\left(X_{s}\right)\left[\psi_{t-s}\right], \quad \psi_{t}=\Lambda^{1 / 2} W_{t} \Lambda^{-1 / 2} \psi \in \mathcal{H} \quad \forall \psi \in \mathcal{H},
$$

где $A \in \operatorname{CPn}(\mathcal{H}), A_{*}(B)[\psi]=\sum_{k}\left(\Phi_{k} \psi, B \Phi_{k} \psi\right)$ (т.е. в силу теоремы Крауза [8] $A$ - ограниченное нормальное вполне положительное отображение), заведомо принадлежит $L_{1}^{\text {loc }}\left(\mathbb{R}_{+}\right)$по переменной $s$ и непрерывно по $t$. Последовательность вполне положительных отображений $P_{t}^{(n)}, n=0,1, \ldots$,

$$
\begin{gathered}
P_{t}^{(0)}(B)=W_{t}^{*} B W_{t} \stackrel{\text { def }}{=} V_{t}(B), \\
P_{t}^{(n+1)}(B) \stackrel{\text { def }}{=} V_{t}(B)+\int_{0}^{t} d s V_{t-s} \Phi P_{s}^{(n)}(B)= \\
=V_{t}(B)+\sum_{k=1}^{n+1} \int_{0}^{t} d s_{1} V_{t-s_{1}} \Phi \ldots \int_{0}^{s_{k-1}} d s_{k} V_{s_{k-1}-s_{k}} \Phi V_{s_{k}-\tau}(B),
\end{gathered}
$$

корректно определена как последовательность ограниченных операторов, соответствующая последовательности квадратичных форм, определенных на $W$-инвариантном плотном множестве $\operatorname{dom} \Lambda^{1 / 2} \subset \operatorname{dom} \Lambda$ и равномерно ограниченных по норме $\mathcal{H}$. Действительно, поскольку операторы $W_{t, s}$ сжимающие, то $0 \leqslant V_{t}(I) \leqslant I$, и из тождества

$$
I-V_{t}(I)=\int_{0}^{t} d s \frac{\partial}{\partial s} V_{t-s}=\int_{0}^{t} d s V_{t-s} \Phi(I)=-\int_{0}^{t} d s \frac{\partial}{\partial s} V_{s}=\int_{0}^{t} d s V_{s} \Phi(I),
$$

справедливого в силу симметричности оператора $H$ на плотном множестве $\operatorname{dom} \Lambda \subseteq$ $\operatorname{dom} \Lambda^{1 / 2}$, следует, что

$$
0 \leqslant \int_{0}^{t} d s V_{t-s} \Phi(I)=\int_{0}^{t} d s V_{s} \Phi(I) \leqslant I
$$

Очевидно, что $P_{t}^{(0)}(B) \leqslant\|B\| I$. Поэтому с помощью соотношений $(6),(7)$ индукцией по $n$ получаем

$$
P_{t}^{(n+1)}(B) \leqslant\|B\|\left(V_{t}(I)+\int_{0}^{t} d s V_{t-s} \Phi(I)\right)=\|B\| I
$$


для любого эрмитова оператора $B$. Таким образом, последовательность вполне положительных отображений $P_{t}^{(n)}$ монотонно возрастает с увеличением $n$ и является равномерно ограниченной: $\left\|P_{t}^{(n)}(B)\right\| \leqslant\|B\|$. Поэтому сушествует наименьшая верхняя грань (l.u.b.)

$$
P_{t}^{\min }(B)=\text { l.u.b. } P_{t}^{(n)}(B),
$$

которая в силу нормальности отображения $\Phi$ удовлетворяет интегральному уравнению

$$
P_{t}^{\min }(B)=V_{t}(B)+\int_{0}^{t} d s V_{t-s} \Phi P_{s}^{\min }(B) .
$$

В случае ограниченных коэффициентов $H$ и $\Phi$ это уравнение эквивалентно дифференциальному уравнению (1) и поэтому называется интегральным уравнением марковской эволюиии. Конструкция минимального решения аналогична конструкции минимального решения для уравнений Колмогорова-Феллера; существование верхней грани и ее минимальные свойства подробно обсуждались в работах [1]-[3], [6].

Заметим далее, что полугруппа $W_{t}$ оставляет инвариантным пространство $\mathcal{H}_{2}$, поэтому оператор $V_{t-s}(\Lambda)$ корректно определен как оператор, соответствующий замыканию положительно определенной квадратичной формы $\left\|\Lambda^{1 / 2} W_{t-s} \psi\right\|^{2}$ на $\mathcal{H}_{2}$. По этой же причине очевидно, что из неравенства (4) следует оценка

$$
\frac{d}{d t}\left\|\Lambda^{1 / 2} W_{t} \psi\right\|^{2}=-2 \operatorname{Re}\left(\Lambda^{1 / 2} G_{t} W_{t} \psi, \Lambda^{1 / 2} W_{t} \psi\right) \leqslant c\left\|\Lambda^{1 / 2} W_{t} \psi\right\|^{2} .
$$

Решая задачу Коши для приведенного выше дифференциального неравенства, мы получаем оценку $V_{t}(\Lambda)_{*}[\psi] \leqslant e^{c t}(\Lambda)_{*}[\psi]$. Следовательно, оператор $P_{t}^{(0)}(\Lambda)$, отвечающий квадратичной форме $\left\|\Lambda^{1 / 2} W_{t} \psi\right\|^{2}$, удовлетворяет неравенству

$$
P_{t}^{(0)}(\Lambda)=W_{t}^{*} \Lambda W_{t}=V_{t}(\Lambda) \leqslant \Lambda e^{c t} .
$$

Предположим, что $P_{t}^{(n)}(\Lambda)_{*}[\psi] \leqslant e^{c t}\left\|\Lambda^{1 / 2} \psi\right\|^{2}$ на $\operatorname{dom} \Lambda$, и докажем аналогичное неравенство для $P_{t}^{(n+1)}(\Lambda)$ по индукции. Из рекуррентного определения $P_{t}^{(n+1)}(\Lambda)$ и предположения (4) имеем

$$
\begin{aligned}
P_{t}^{(n+1)}(\Lambda)_{*}[\psi] \stackrel{\text { def }}{=} V_{t}(\Lambda)_{*}[\psi]+\sum_{k} \int_{0}^{t} d s \sum_{k} P_{s}^{(n)}(\Lambda)_{*}\left[\Phi_{k} W_{t-s} \psi\right] \leqslant \\
\leqslant V_{t}(\Lambda)_{*}[\psi]+\int_{0}^{t} d s e^{c s} \sum_{k} \Lambda_{*}\left[\Phi_{k} W_{t-s} \psi\right]= \\
=\Lambda_{*}\left[W_{t} \psi\right]+\int_{0}^{t} d s e^{c s} \sum_{k}\left\|\Lambda^{1 / 2} \Phi_{k} W_{t-s} \psi\right\|^{2} \leqslant \\
\leqslant \Lambda_{*}\left[W_{t} \psi\right]+\int_{0}^{t} d s e^{c s}\left(c \Lambda_{*}\left[W_{t-s} \psi\right]+\right. \\
\left.\quad+2 \operatorname{Re}\left(\Lambda^{1 / 2} G W_{t-s} \psi, \Lambda^{1 / 2} W_{t-s} \psi\right)\right)= \\
=\Lambda_{*}\left[W_{t} \psi\right]+\int_{0}^{t} d s \frac{\partial}{\partial s}\left(e^{s c}\left\|\Lambda^{1 / 2} W_{t-s} \psi\right\|^{2}\right)=\left\|\Lambda^{1 / 2} \psi\right\|^{2} e^{c t},
\end{aligned}
$$


где все неравенства понимаются в смысле неравенств для замыкаемых квадратичных форм, корректно определенных на $\operatorname{dom} \Lambda$ и непрерывно зависящих от $s$. Отсюда следует оценка, указанная в формулировке теоремы. Теорема доказана.

Пусть $T_{t}$ - квантовая динамическая полугруппа, двойственная к $P_{t}^{\min }$ относительно билинейной формы $\langle\rho, B\rangle=\operatorname{Tr} \rho B$. Из оценки (5) следует, что для любого оператора $B$ такого, что $\left\|\Lambda^{-1 / 2} B \Lambda^{-1 / 2}\right\|<\infty$, выполнена оценка

$$
\begin{aligned}
\operatorname{Tr}\left\{B T_{t}(\rho)\right\} & =\operatorname{Tr}\left\{\left(\Lambda^{-1 / 2} B \Lambda^{-1 / 2}\right)\left(\Lambda^{1 / 2} T_{t}(\rho) \Lambda^{1 / 2}\right)\right\} \leqslant \\
& \leqslant\left\|\Lambda^{-1 / 2} B \Lambda^{-1 / 2}\right\| \operatorname{Tr}\left\{P_{t}^{\min }(\Lambda) \rho\right\} \leqslant e^{c t}\left\|\Lambda^{-1 / 2} B \Lambda^{-1 / 2}\right\| \operatorname{Tr}\left\{\Lambda^{1 / 2} \rho \Lambda^{1 / 2}\right\} .
\end{aligned}
$$

Эта оценка означает, что при условии (4) сушествует подалгебра операторов с конечным следом $\rho=\Lambda^{-1 / 2} \sigma \Lambda^{-1 / 2}, \sigma \in \mathcal{T}(\mathcal{H})$, инвариантная относительно разрешаюшего оператора квантового уравнения марковской эволюции $T_{t}$. С другой стороны, в работах [3], [7] показано, что наличие оценки (5) гарантирует консервативность минимальной КДП (т.е. $\left.P_{t}(I)=I \forall t>0\right)$ и единственность этого решения в классе консервативных КДП.

Благодарности. Работа вьполнена при поддержке INTAS (грант № 99-00545).

\section{Список литературы}

[1] E. B. Davies. Rep. Math. Phys. 1979. V. 11. № 2. P. 169.

[2] A.S. Holevo. Stochastic differential equations in Hilbert space and quantum Markovian evolutions. In: Probability Theory and Mathematical Statistics. Proc. of the Seventh Japan-Russian Symposium. Eds. S. Watanabe et al. Singapore: World Scientific, 1996. P. 122.

[3] A. M. Chebotarev, F. Fagnola. J. Funct. Anal. 1998. V. 153. № 2. P. 382.

[4] R. Schack, T. A. Brun, I. C. Percival. Phys. Rev. A. 1996. V. 53. P. 2694.

[5] R. Schack, T. A. Brun. Comput. Phys. Commun. 1997. V. 102. № 1-3. P. 210.

[6] Т. Като. Теория возмушений линейных операторов. М.: Мир, 1972.

[7] A. M. Chebotarev, J. C. Garcia, R. B. Quezada. RIMS Kokyuroku. 1998. V. 1035. P. 44.

[8] K. Kraus. Ann. Phys. 1971. V. 64. P. 311. 